\title{
CORRIGENDUM
}

\section{Non-standard real-analytic realizations of some rotations of the circle - CORRIGENDUM}

\author{
SHILPAK BANERJEE
}

doi:10.1017/S0143385715001108, Published by Cambridge University Press, 18 April 2016.

Abstract. We correct two technical errors in the original paper. The main result in the original paper remains valid without any changes.

We would like to correct two errors in [1], the first of which was repeated in [2].

(i) The complexification of the function $s_{\alpha, N}$ used to approximate the 'step' function $\tilde{s}_{\alpha, N}$ defined in the proof of Lemma 4.7 need not converge. Instead one can use either a Fourier series approximation or the following function (suggested by Philipp Kunde) with the additional assumption that $k$ is even:

$$
\begin{aligned}
s_{\alpha, N}(x):= & \left(\sum_{i=0}^{k / 2-1} \alpha_{i}\left(e^{-e^{-A \sin (2 \pi(N x-i / k))}}-e^{\left.-e^{-A \sin (2 \pi(N x-(i+1) / k))}\right)}\right) e^{-e^{-A \sin (2 \pi N x)}}\right. \\
& +\left(\sum_{i=k / 2}^{k-1} \alpha_{i}\left(e^{-e^{-A \sin (2 \pi(N x-i / k))}}-e^{-e^{-A \sin (2 \pi(N x-(i+1) / k))}}\right)\right) e^{-e^{A \sin (2 \pi N x)}} .
\end{aligned}
$$

Note that in addition to all the requirements of Lemma 4.7, the function $s_{\alpha, N}$ in $(0.1)$ satisfies the derivative condition $\sup _{x \in[0,1) \backslash F}\left|s_{\alpha, N}^{\prime}(x)\right|<\varepsilon$ required in [2].

(ii) Proposition 5.1 claims more than what we can prove. It should be replaced by the following version.

Proposition 5.1. Fix any $\rho>0$. Then, for an appropriately chosen sequence $k_{n}$, we have $T_{n} \rightarrow T$ for some $T \in$ Diff ${ }_{\rho}^{\omega}\left(\mathbb{T}^{2}\right)$. (We cannot guarantee that the complexification of the lift ( to $\mathbb{R}^{2}$ ) of $T$ can be holomorphically extended to the whole of $\mathbb{C}^{2}$, but it is easy to see that it extends to a fixed strip in $\mathbb{C}^{2}$ containing $\mathbb{R}^{2}$.)

Proof. Let $\varepsilon>0$ and let $\varepsilon_{n}$ be a sequence such that $\sum_{n=1}^{\infty} \varepsilon_{n}<\varepsilon$. Now notice that with $d_{\rho}$ denoting the usual distance in Diff ${ }_{\rho}^{\omega}\left(\mathbb{T}^{2}\right)$, we have $d_{\rho}\left(T_{n+1}, T_{n}\right)=d_{\rho}\left(H_{n+1}^{-1} \circ\right.$ $\left.\phi^{\alpha_{n+1}} \circ H_{n+1}, H_{n}^{-1} \circ \phi^{\alpha_{n}} \circ H_{n}\right)=d_{\rho}\left(H_{n+1}^{-1} \circ \phi^{\alpha_{n}} \circ \phi^{1 / k_{n} l_{n}^{2} q_{n}} \circ H_{n+1}, H_{n}^{-1} \circ \phi^{\alpha_{n}} \circ H_{n}\right)$ $=d_{\rho}\left(H_{n}^{-1} \circ \phi^{\alpha_{n}} \circ h_{n+1}^{-1} \circ \phi^{1 / k_{n} l_{n}^{2} q_{n}} \circ H_{n+1}, H_{n}^{-1} \circ \phi^{\alpha_{n}} \circ H_{n}\right)<\varepsilon_{n}$. The last step is guar- 
anteed after choosing a large enough $k_{n}$. This is possible because the construction of $h_{n+1}$ does not involve $k_{n}$. So, we are free to make it as large as we want.

\section{REFERENCES}

[1] S. Banerjee. Non-standard real-analytic realizations of some rotations of the circle. Ergod. Th. \& Dynam. Sys., doi:10.1017/etds.2015.110.

[2] P. Kunde. Real-analytic weak mixing diffeomorphisms preserving a measurable Riemannian metric. Ergod. Th. \& Dynam. Sys., accepted. 